\title{
Cerebral Metabolism in Man after Acute Stroke: New Observations Using Localized Proton NMR Spectroscopy
}

\author{
H. Bruhn, J. Frahm, M. L. Gyngell, K. D. Merboldt, W. Hänicke, \\ AND R. SAUTER* \\ Max-Planck-Institut für biophysikalische Chemie, Postfach 2841, D-3400 Göttingen, \\ Federal Republic of Germany; and ${ }^{*}$ Bereich Medizinische Technik, Siemens AG, \\ Henkestrasse 127, D-8520 Erlangen, Federal Republic of Germany
}

Received August 26, 1988

\begin{abstract}
Localized proton NMR spectroscopy at $1.5 \mathrm{~T}$ using stimulated echoes has been applied to study metabolic alterations in the postischemic phase of patients with acute cerebral infarction. A complete depletion of $\mathrm{N}$-acetyl aspartate in the area of infarction has been observed in a patient studied 4 days after stroke. This finding was paralleled by a dramatic increase in the concentration of lactic acid to about $16 \mathrm{mM}$ within the lesion, indicating continued anaerobic glycolysis. The diluting effect of the edema has been estimated to reduce average metabolite concentrations by about a factor of 3 . c) 1989 Academic Press, Inc.
\end{abstract}

\section{INTRODUCTION}

The direct and noninvasive observation of metabolic consequences of stroke in man has hitherto not been possible. Although many attempts have been made to come as close as possible to in vivo conditions in destructive animal studies, major difficulties and insecurities of classical analytical methods that depend on tissue freezing arise from rapid and unknown changes in brain metabolism postmortem. Previous proton NMR studies on animals concentrated only on the observation of a rise of cerebral lactate under ischemic conditions or postmortem. Since these approaches were based on invasive applications of surface coils combined with spectral difference techniques they are not applicable to human studies and are further complicated by a lack of reliable localization and spectral resolution. Here we report an initial application of the stimulated echo (STEAM) method $(1,2)$ for image-selected localized proton NMR spectroscopy to the evaluation of an ischemic brain lesion in a patient 4 days after acute infarction.

\section{METHODS}

Localization with the STEAM sequence is achieved by excitation of three orthogonal slices using selective radiofrequency ( $\mathrm{rf}$ ) pulses applied in the presence of orthogonal magnetic field gradients

$$
\text { 90 (CHESS)-90 (\#1)-TE/2-90 (\#2)-TM-90 (\#3)-TE/2-STE. }
$$

The stimulated echo (STE) at echo time TE originates only from spins (protons) at the intersection of these slices. Water suppression is accomplished by preceding 
chemical-shift selective (CHESS) rf pulses. For human investigations the STEAM sequence has been implemented on a conventional 1.5-T MRI system (Siemens Magnetom) (2). The parameters where TM $=30 \mathrm{~ms}, \mathrm{TE}=50$ and $270 \mathrm{~ms}$, TR $=1500$ $\mathrm{ms}$, and 512 acquisitions to obtain sufficient signal-to-noise from a $27-\mathrm{ml}(3 \times 3 \times 3$ $\left.\mathrm{cm}^{3}\right)$ volume-of-interest (VOI). Multislice FLASH MR images $(256 \times 256$ pixels, TR $=100 \mathrm{~ms}, \mathrm{TE}=11 \mathrm{~ms}, \alpha=70^{\circ}$ ) were recorded in all three orientations to determine the position and size of the VOI (3).

The 42-year-old patient presented here suffered from diffuse arteriosclerosis and had a history of heavy cigarette smoking. Five months earlier CT investigations indicated an occlusion within both anterior cerebral arteries. Four days prior to this investigation the patient had been hospitalized due to an acute stroke with resulting aphasia and right hemiparesis. CT showed an extensive ischemic lesion in the left frontodorsal and insular area indicating an infarct of the left medial cerebral artery. Prior to examination informed consent had been obtained.

\section{RESULTS}

Figure 1 shows typical proton NMR spectra from a $27-\mathrm{ml}$ VOI in the insular area of a normal control at $\mathrm{TE}=50 \mathrm{~ms}$ (a) and $\mathrm{TE}=270 \mathrm{~ms}$ (b), respectively. Major resonances include residual mobile lipids (Lip, 1.0-1.5 ppm), acetate (Ac, $1.85 \mathrm{ppm}$ ), $N$-acetyl aspartate (NAA, 2.0 and $2.6 \mathrm{ppm}$ ), $\gamma$-amino butyric acid (GABA), glutamine (Gln, $2.45 \mathrm{ppm}$ ), aspartate (Asp, $2.8 \mathrm{ppm}$ ), creatine and phosphocreatine $(\mathrm{Cr} / \mathrm{PCr}$, 3.0 and $3.9 \mathrm{ppm}$ ), choline-containing compounds (Cho, $3.2 \mathrm{ppm}$ ), and inositols (Ins, $3.5 \mathrm{ppm})(2,4)$. The resonance at $3.75 \mathrm{ppm}$ originates from $\alpha-\mathrm{CH}$ groups within various amino acids. The spectrum in Fig. $1 \mathrm{~b}$ at $\mathrm{TE}=270 \mathrm{~ms}$ is simplified due to the short $T_{2}$ relaxation times of some resonances (4). In particular, the resonance signals of residual mobile lipids have completely vanished at this echo time. Since the normal concentration of cerebral lactate was found to be in the range below $1 \mathrm{mM}(4)$, its resonance signal is not observed because of the limited signal-to-noise ratio of a spectrum from a $27-\mathrm{ml}$ VOI.

Figure 2 depicts axial (a) and coronal (b) FLASH NMR images of the brain of the patient 4 days after presenting with an acute stroke in the left frontodorsal area. The VOI selected for localized proton spectroscopy is indicated by rectangles.

The patient spectra in Fig. 3 at TE $=50 \mathrm{~ms}$ (a) and TE $=270 \mathrm{~ms}(\mathrm{~b})$, respectively, display striking differences from the normal control. Signal intensities of both spectra are multiplied by a factor of 2 compared to Fig. 1. While the NAA resonance has completely disappeared, the strong doublet at $1.3 \mathrm{ppm}$ is due to the $\mathrm{CH}_{3}$ group of lactic acid. Moreover, in the patient spectrum at $\mathrm{TE}=50 \mathrm{~ms}$, choline and creatine resonance signals are reduced by factors of 3 and 5, respectively. Accordingly, the $\mathrm{Cho} / \mathrm{Cr}$ ratio changed from 0.6 in the control to 1.0 in the infarct. Resonances from acetate and aspartate are not present in the patient spectra, while small unresolved resonances in the range from 2.1 to $2.35 \mathrm{ppm}$ may be due to residual contributions from glutamate and glutamine.

\section{DISCUSSION}

A translation of the dramatic changes observed in the proton spectrum of the 4day-old brain infarct into alterations of metabolite concentrations must take into 


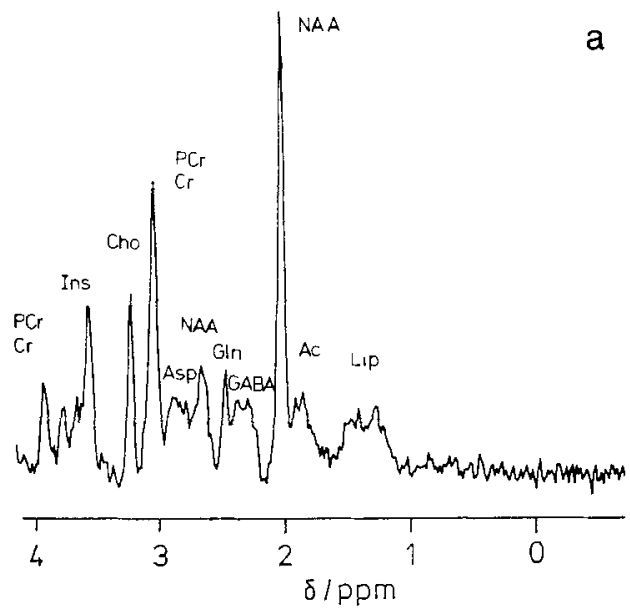

FIG. 1. Sixty-four-megahertz (1.5-T) proton NMR spectra of normal human brain from a $3 \times 3 \times 3-\mathrm{cm}^{3}$ VOl localized in the insular area of a healthy volunteer using the STEAM sequence (TM $=30 \mathrm{~ms}$, TR $=1500 \mathrm{~ms}, 512$ scans). Spectra were recorded at (a) $\mathrm{TE}=50 \mathrm{~ms}$ and (b) $\mathrm{TE}=270 \mathrm{~ms}$. Intensities are directly comparable. Resonance signals of major metabolites are due to residual lipids (Lip), acetate (Ac), $N$-acetyl aspartate (NAA), $\gamma$-amino butyric acid (GABA), glutamine (Gln), aspartate (Asp), creatine/phosphocreatine ( $\mathrm{Cr} / \mathrm{PCr}$ ), choline-containing compounds (Cho), and inositols (Ins).

account the diluting effect of the accompanying brain edema. Being predominantly of an intracellular nature (5) swelling of the brain will ensue within minutes after onset of ischemia due to the breakdown of energy-dependent osmoregulatory processes within the membranes (e.g., $\mathrm{Na}^{+}-\mathrm{K}^{+}$exchange pump) of both cerebral parenchyma and vascular endothelium (blood-brain barrier). Lysosomal enzymatic digestion of structural elements may further aggravate the edema within a few hours. In the proton spectra the reduction of the choline resonance signal allows an estimate of the dilutional effect on average concentrations computed from the $27-\mathrm{ml}$ VOI. If we assume that the $\mathrm{N}-\left(\mathrm{CH}_{3}\right)_{3}$ groups within the choline-containing compounds are resistant to ischemic degradation, then its threefold reduction at $\mathrm{TE}=50 \mathrm{~ms}$ (Figs. la and 3a) must be attributed to the edema. On the other hand, the reduction factor of 5 observed for creatine (all phosphocreatine being split to creatine) would thus translate into an additional loss of about $40 \%$ in total creatine concentration by a degradative or leaking-out mechanism.

In assuming an average concentration of $10.5 \mathrm{~m} M$ for total creatine in normal brain (6) and using $T_{1}$ and $T_{2}$ relaxation times of lactate as previously determined (4), the massive increase in the $\beta-\mathrm{CH}_{3}$ doublet resonance signal from lactate refers to an average concentration of about $16 \mathrm{~m} M$ within the ischemic lesion. Although necrosis of a large number of cells would be expected to prevail in a 4-day-old infarct, this finding clearly indicates that anaerobic glycolysis is continuously present to a surprisingly high degree. It further supports the widely held view (7) that focal cerebral ischemia can rarely be regarded as complete but enables partial substrate delivery such as glucose in a hypoperfusion condition. No conclusions about the microheterogeneity within the lesion can be drawn from $27-\mathrm{ml}$ VOI measurements. 


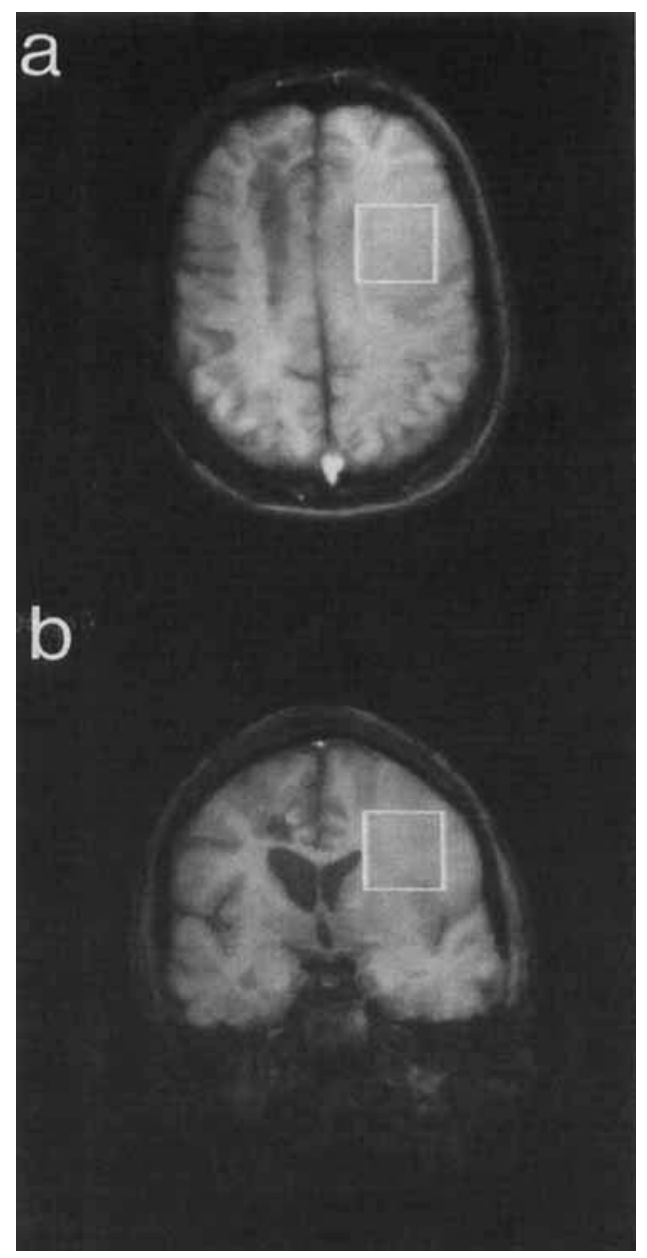

FIG. 2. Sixty-four-megahertz (1.5-T) axial (a) and coronal (b) FLASH NMR images of the brain of a 42 year-old patient 4 days after presenting with an acute stroke in the left frontodorsal area $(\mathrm{TR}=100 \mathrm{~ms}$, $\mathrm{TE}=10 \mathrm{~ms}, \alpha=70^{\circ}$ ). The rectangles indicate the $3 \times 3 \times 3-\mathrm{cm}^{3}$ VOI selected for localized proton NMR spectroscopy.

In addition to the increase in cerebral lactate, the disappearance of NAA represents another hallmark of the infarct spectrum. While the edema within the ischemic lesion may explain the loss of resonance signals from metabolites in the 1-2 $\mathrm{m} M$ range, e.g., acetate, GABA, and aspartate, it does not account for the decrease in the NAA resonance signal to below detectability. In fact, this is a remarkable observation because it differs from the high degree of stability of the NAA pool found in various experimental conditions $(8)$. It is therefore plausible to assume an active degradative metabolic mechanism for NAA that occurs in injured neurons already in the first days (possibly hours) after onset of infarct. It may be hypothesized that amidohydrolase ( 9 ) which is thought to be compartmentalized or membrane-bound in healthy nervous 


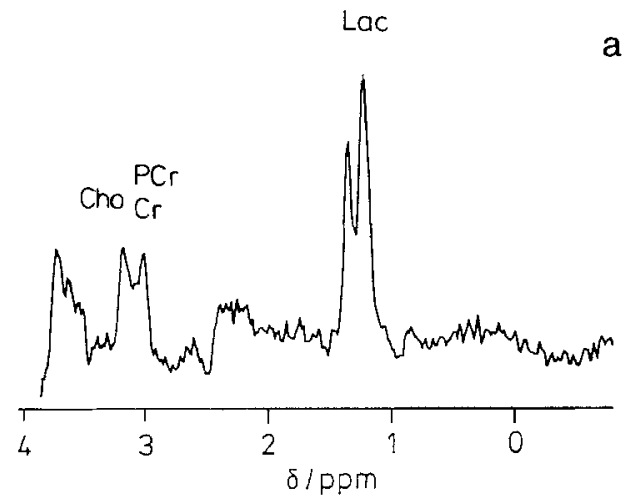

a

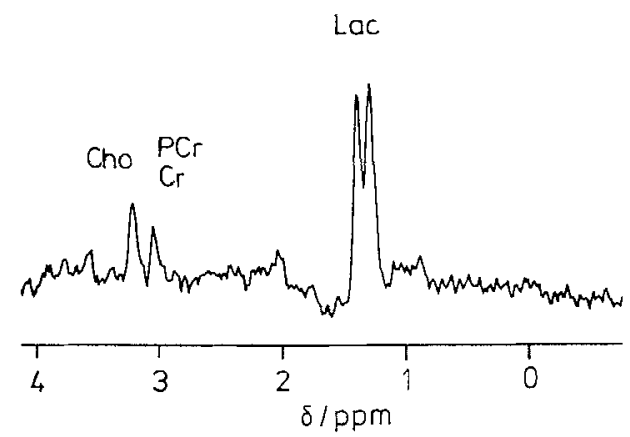

FIG. 3. Sixty-four-megahertz (1.5-T) proton NMR spectra localized in the area of infarction as indicated in the images of the patient in Fig. 2 using the STEAM sequence $(27 \mathrm{ml}, \mathrm{TM}=30 \mathrm{~ms}, \mathrm{TR}=1500 \mathrm{~ms}, 512$ scans). Spectra were recorded at (a) $\mathrm{TE}=50 \mathrm{~ms}$ and (b) $\mathrm{TE}=270 \mathrm{~ms}$. Intensities are multiplied by a factor of 2 compared to Fig. 1 . Note the complete absence of a resonance signal from $N$-acetyl aspartate and the strong contribution from lactic acid (Lac). Creatine/phosphocreatine $(\mathrm{Cr} / \mathrm{PCr}$ ) and choline-containing compounds (Cho) are reduced by factors of 5 and 3, respectively, compared to normal control (Fig. 1).

tissue (10) may be released or activated in neuronal cell death in vivo. This is supported by a report that the NAA concentration decreased only very slowly in whole pieces of monkey brain, whereas it declined very rapidly (within $2 \mathrm{~h}$ ) in incubated brain homogenates $(11)$. Other studies looking for excitatory neurotransmitters employed pathway-specific lesions in rat brain and observed a $40-60 \%$ decrease in NAA in dependent neurons within a few days (12). Further work is in progress to explore changes in postischemic metabolite levels in vivo in various stages after acute infarction.

\section{CONCLUSIONS}

Metabolic alterations in a patient after acute cerebral infarct include a complete loss of $N$-acetyl aspartate and a dramatic increase in lactic acid. It may be concluded that prolonged focal cerebral ischemia in man is accompanied by a breakdown of the neuronal NAA stores within a few days after infarct. This finding appears to be diagnostic for neuronal cell death.

The possibility of a direct and noninvasive monitoring of focal postischemic metabolism in man is expected to mark a milestone in clinical research on stroke. It will contribute to a better understanding of neuronal injury as well as facilitating the control and development of stroke therapy. In addition to cell studies and animal models, the present findings emphasize the importance of noninvasive biochemical studies in vivo which may now be extended by localized proton NMR spectroscopy in man using conventional MRI systems operating at field strengths of only $1.5 \mathrm{~T}$.

\section{ACKNOWLEDGMENTS}

Financial support by the Bundesminister für Forschung und Technologie (BMFT) of the Federal Republic of Germany (Grant 01 VF 8606/6) is gratefully acknowledged. We thank Dr. Schuierer and the clinicians from the Department of Neurology, University of Erlangen, for allowing us to study their patients. 


\section{REFERENCES}

1. J. Frahm, K. D. Merboldt, AND W. HÄNICKE, J. Magn. Reson. 72, 502 (1987).

2. J. Frahm, H. BruhN, M. L. Gyngell, K. D. Merboldt, W. HäniCKE, AND R. SAUter, Magn. Reson. Med. 9, 79 (1989).

3. J. FRAHM, Naturwissenschaften 74, 415 (1987).

4. J. Frahm, H. Bruhn, M. L. Gyngell, K. D. Merboldt, W. Hänicke, and R. Sauter, Magn. Reson. Med., in press.

5. T. Fujimoto, J. T. WalkeR, M. SPATZ, AND I. Klatzo, in "Dynamics of Brain Edema" (H. M. Pappius and W. Feindel, Eds.), p. 171 ff., Springer-Verlag, Berlin, 1976.

6. L. D. Lewis, B. LJUNGGren, R. A. Ratcheson, AND B. K. SieSJö, J. Neurochem. 23, 673 (1974).

7. L. G. SAlford, F. Plum, AND B. K. SiEsJö, Arch. Neurol. 29, 227 (1973).

8. K. B. JaCOBSON, J. Gen. Physiol. 43, 323 (1959).

9. A. F. D'Adamo, J. Peisach, G. Manner, and C. T. Weiler, J. Neurochem. 28, 739 (1977).

10. F. B. GoldDSteIn, J. Neurochem. 26, 45 (1976).

11. F. H. Bruns, H. Reinauer, AND W. STORK, Hoppe-Seyler's Z. Physiol. Chem. 348, 512 (1967).

12. K. J. Koller, R. ZACZEK, AND J. T. COYLE, J. Neurachem. 43, 1136 (1984). 